\title{
Nutritional yeast culture has specific anti-microbial properties without affecting healthy flora. Preliminary results*
}

\author{
G.S. Jensen ${ }^{1,4}$, K.M. Patterson ${ }^{2}$ and I. Yoon ${ }^{3}$ \\ ${ }^{1}$ Holger NIS Inc., 601 13th Avenue NE, Calgary, Alberta, T2E 1C7, Canada \\ ${ }^{2}$ NIS Labs, 1437 Esplanade, Klamath Falls, OR 97601, USA \\ ${ }^{3}$ Diamond V Mills, 838 1st Street NW, Cedar Rapids, IA 52407, USA
}

(Received 19 June 2007; revised version 23 January 2008; accepted 8 April 2008)

\begin{abstract}
The objective of this study was to perform a preliminary assessment of the potential antimicrobial effects in vitro of nutritional yeast culture (Diamond V XPTM), containing Saccharomyces cerevisiae, media on which it was grown, and metabolites produced during fermentation. It was tested against Escherichia coli ATCC25922, Staphylococcus aureus ATCC25923, Candida tropicalis ATCC13803, and oral flora from human saliva. Serial dilutions of each microbe were mixed with either water or an aqueous extract of yeast culture, and plated on aerobic, coliform, or yeast $/$ mold Petrifilm $^{\mathrm{TM}}$, respectively. Colony counts and appearances showed that yeast culture induced strong inhibition of $C$. tropicalis growth, moderate inhibition of $E$. coli, and only minimal effect on growth of $S$. aureus or oral flora. Metabolic activity of $E$. coli was affected, as seen by reduced beta-glucuronidase activity and lack of gas formation indicative of lactose fermentation. The data suggest that yeast culture consumption may support beneficial composition of intestinal flora while promoting containment of some microbial species.
\end{abstract}

KEY WORD: nutritional yeast culture, Saccharomyces cerevisiae, Escherichia coli, Candida tropicalis, normal flora, antimicrobial

\section{INTRODUCTION}

Enterotoxigenic Escherichia coli-associated diarrhoea represents one of the major threats to health and survival among farm animals. Escalated antibiotic re-

\footnotetext{
* Supported by Diamond V Mills Inc., Cedar Rapids, Iowa

${ }^{4}$ Corresponding author: e-mail: gitte@holgernis.com
} 
sistance is a major concern, not only with regards to economical loss to farmers world-wide but also for associated human health hazards. Intestinal flora in animals and humans constitute a reservoir of resistance genes, or potentially pathogenic bacteria (van den Bogaard and Stobberingh, 2000). Transfer of antibiotic resistance genes between commensal and pathogenic organisms includes transfer of antimicrobial resistance from potentially pathogenic bacteria in farm animals to commensal organisms in human intestinal flora via consumed meat products (Witte, 2000).

Once an animal is compromised by pathogenic enteric bacteria such as some forms of $E$. coli, other opportunistic infections can further contribute to the deterioration of the animal. Examples include infections with Candida subspecies in post-weaning multisystemic wasting syndrome in pigs (Zlotowski et al., 2006) and, increasingly, mastitis among dairy cows in areas of Africa (Kivaria and Noordhuizen, 2006). In addition, Candida subspecies may also be primary causes of diarrhoea in newborn calves (Elad et al., 1998).

Dietary strategies involve feed supplements such as yeast culture, which is used as a feed additive in all types of farm animals and has been shown to increase milk production (Dann et al., 2002) and feed efficiency in dairy cows (Schingoethe et al., 2004), weaning weight of pigs (Kim et al., 2006), and egg production in chickens (Tangendjaja and Yoon, 2002). In addition, yeast culture increases the ratio of lactobacilli over coliform bacteria in laying hens (Liu et al., 2002). Therefore, the objective of this preliminary study was to evaluate evidence for specific antimicrobial effects of yeast culture in vitro.

\section{MATERIAL AND METHODS}

Three different types of Petrifilm ${ }^{\mathrm{TM}}$ were purchased from 3M Microbiology: aerobic culture (AC), Escherichia coli/coliform (EC), and yeast and mold (YM) plates. Each Petrifilm plate is a sample-ready, self-contained, self-sealing system, and manufacturer's protocols for each plate are validated methods by AOAC International. Each plate consists of a counting surface with a grid of $1 \mathrm{~cm}^{2}$ fields, pre-coated with microbe-specific nutrients, a cold-water soluble gelling agent, and precursor dyes. These dyes are transformed into colour indicators by microbial enzyme processes and help to visualize microbial colonies upon incubation.

An extract containing soluble metabolites from the yeast culture was prepared in the following 3-step protocol: 1 . An amount of $0.5 \mathrm{~g}$ yeast culture (Diamond V XPTM Yeast Culture, Diamond V Mills) was weighed and added to $5 \mathrm{ml}$ phosphate-buffered saline (PBS, Sigma-Aldrich). This mixture was vortexed for 30 $\mathrm{sec}$ and allowed to sit at room temperature for one h. No homogenization was 
performed to disrupt yeast cells in the product. 2. Remaining solids were removed by centrifugation at $2400 \mathrm{rpm}$ for $10 \mathrm{~min}$ to remove the majority of solids. 3 . The supernatant was harvested and any remaining solids were removed by filtration using a 0.22 micron syringe filter. The resulting extract contained soluble metabolites without yeast cell walls and was orange in colour.

The following microbial strains were obtained from the American Type Culture Collection (ATCC): Escherichia coli ATCC 25922, Staphylococcus aureus ATCC 25923, and Candida tropicalis ATCC 13803. Healthy oral flora was obtained by unstimulated saliva collection from human subjects. Stock solutions of microbes were prepared by rehydration of lyophilized organisms from the ATCC vials into $5 \mathrm{ml}$ sterile water, which was allowed to sit at $4{ }^{\circ} \mathrm{C}$ overnight. Oral flora was obtained by mixing $1 \mathrm{ml}$ unstimulated saliva with $9 \mathrm{ml}$ sterile water. Plating of serial dilutions allowed assessment of numbers of viable organisms per $\mathrm{ml}$. The stock suspension was adjusted to approximately $10^{4}$ viable microbes per $\mathrm{ml}$. Serial, 10-fold dilutions were made from this stock.

Serial dilutions of all 4 microbial suspensions were made in sterile PBS. Each dilution was then mixed in equal proportions with either PBS (control) or yeast culture extract immediately before plating E. coli, S. aureus, oral flora, and $C$. tropicalis onto the appropriate type of Petrifilm (EC, AC, AC, and YM plates, respectively). Escherichia coli/coliform and AC plates were incubated for 24 and $48 \mathrm{~h}$ at $35^{\circ} \mathrm{C}$. Yeast and mold plates were incubated at $25^{\circ} \mathrm{C}$ for up to 6 days. Colonies were counted, and Petrifilm plates were photographed.

\section{RESULTS}

Growth of C. tropicalis in the presence of yeast culture was almost completely undetectable over a 1,000-fold dilution range. Even at the highest concentration of plated $C$. tropicalis, only minor colony formation was observed. These colonies were smaller than colonies on untreated control plates, and only occurred along the edges of the plate (Figure 1, Table 1).

Growth of $E$. coli was reduced in the presence of yeast culture. Even when high concentrations of $E$. coli were plated, colonies that were observed were extremely small compared to colonies on untreated control plates (Figure 2, Table 1 ). There was also evidence that $E$. coli were less metabolically active in the presence of yeast culture. This was indicated by reduction in the indicator dye for beta-glucuronidase activity and reduced metabolic gas formation from lactose fermentation associated with each colony. Gas formation can be seen on Figure 2 (indicated by arrows) at the 1:100 dilution of $E$. coli stock, in the top panel of control cultures. 
Table 1. Colony counts for microbial growth in the absence versus presence of yeast culturea ${ }^{1}$

\begin{tabular}{|c|c|c|c|c|c|c|c|c|}
\hline \multirow{2}{*}{ Dilutions } & \multicolumn{2}{|c|}{ C. tropicalis } & \multicolumn{2}{|c|}{ E. coli } & \multicolumn{2}{|c|}{ S. aureus } & \multicolumn{2}{|c|}{ Oral flora } \\
\hline & control & $\mathrm{XP}$ & control & $\mathrm{XP}$ & control & $\mathrm{XP}$ & control & $\mathrm{XP}$ \\
\hline $1: 1$ & 500 & 0 & $++++^{2}$ & $++^{3}$ & 268 & 175 & ++ & ++ \\
\hline $1: 10$ & 216 & 0 & +++ & + & 114 & 44 & + & + \\
\hline $1: 100$ & 74 & 0 & 14 & $7^{4}$ & 31 & 15 & 158 & 129 \\
\hline $1: 1000$ & 16 & 0 & 0 & 0 & 7 & 9 & 21 & 19 \\
\hline
\end{tabular}

${ }^{1}$ the data shown are representative of three independent tests; ${ }^{2}$ plusses indicate that the colony density was too numerous to count, and that relative growth was estimated; ${ }^{3}$ the $E$. coli colonies growing in the presence of XP were more diffuse, smaller, and less distinct, in part due to a lower amount of beta-glucuronidase activity, as reflected by the lower level of indicator dye associated with the colonies; ${ }^{4}$ these colonies were barely visible due to a low amount of beta-glucuronidase activity. These colonies were not associated with gas bubbles, indicating low or no lactose fermentation activity

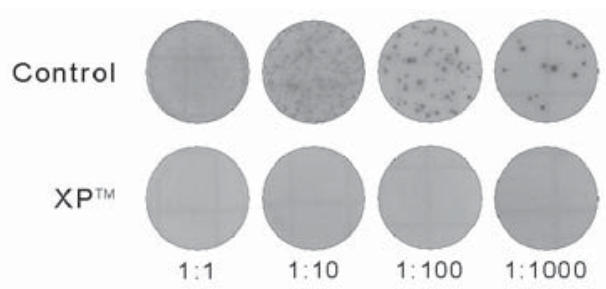

Figure 1. Growth inhibitory effect of yeast culture on Candida tropicalis. Serial dilutions of microbial culture were mixed with an equal amount of yeast culture extract or PBS and plated onto Petrifilm. Colonies appeared within 3 days on untreated film (top row), whereas no colonies were seen on films treated with yeast culture (bottom row). Data shown are representative of three separate experiments

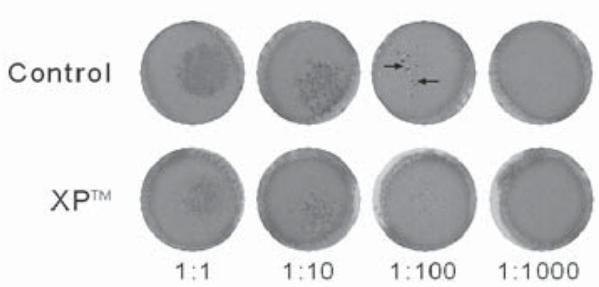

Figure 2. Anti-coliform effect of yeast culture on cultures of Escherichia coli. Serial dilutions of microbial culture were mixed with an equal amount of yeast culture extract or PBS and plated onto Petrifilm. Colonies appeared within $24 \mathrm{~h}$ on both untreated films (top row) and films treated with yeast culture (bottom row). The colonies grown in the presence of yeast extract were more difficult to count, due to their diffuse appearance, lack of metabolic activity as reflected by low amount of indicator dye for beta-glucuronidase activity, lack of gas formation associated with lactose fermentation (see arrows on control plate), and smaller size. Data shown are representative of three separate experiments 
Growth of S. aureus was only mildly affected by inclusion of yeast culture. Growth inhibition of $S$. aureus was only evident at the highest concentrations of plated microbes, where slightly smaller colonies were seen (Figure 3, Table 1). This indicated that a changed profile of essential nutrients as a result of addition of yeast culture, rather than a direct antimicrobial effect, was providing less-than-optimal culture conditions at high population densities with higher competition for nutrients.

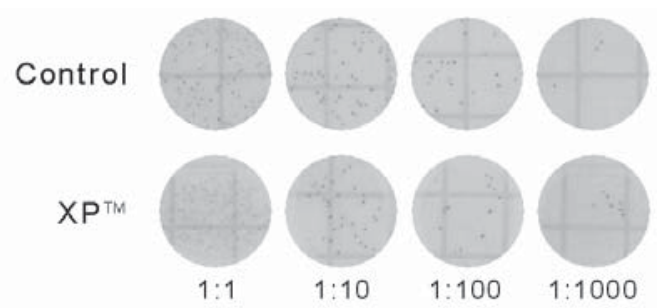

Figure 3. Anti-microbial effect of yeast culture on cultures of Staphylococcus aureus. Serial dilutions of microbial culture were mixed with an equal amount of yeast culture extract or PBS and plated onto Petrifilm. Colonies appeared within $24 \mathrm{~h}$. Almost no difference was seen between untreated (top row) and yeast culture-treated (bottom row) cultures. Data shown are representative of three separate experiments

As a control, healthy oral flora was used as a mixed source of predominantly commensal organisms. Growth of oral bacteria from healthy saliva samples were almost unaffected by the addition of yeast culture (Table 1).

\section{DISCUSSION}

Yeast culture is a Saccharomyces cerevisiae based product that is used as a nutritional additive to animal feed (Schingoethe et al., 2004; Barnes et al., 2006). Data presented here show that XP yeast culture specifically inhibited growth of $E$. coli and C. tropicalis, with only a minor inhibition of growth of $S$. aureus or healthy oral flora. The effect was dose-dependent. It was estimated that XP yeast culture inhibited the growth of $C$. tropicalis 1,000-fold, while also inhibiting the growth as well as the metabolic activity of $E$. coli. This selective anti-microbial effect is promising, as it supports the argument that consumption of XP yeast culture may help reduce harmful coliform bacteria and fungal organisms such as Candida subspecies, while not causing an imbalance of healthy intestinal flora.

Specific anti-microbial effects may be contributing factors to the previously observed effects of yeast culture in vivo, as the support of commensal organisms while containing potentially pathogenic organisms is particularly important around the time of weaning, when protective effects of colostrum cease and the animal is only beginning to develop immunity. There may be very little time and chance for vaccination to provide adequate protection, and dietary compounds such as yeast culture may offer protective effects during this period. 


\section{CONCLUSIONS}

Based on these studies in vitro, XP yeast culture has a modulating effect on microbial growth, favouring some aerobic bacteria while inhibiting the growth and metabolism of E. coli and C. tropicalis. To what extent this extends to other coliform and fungal microorganisms remains to be tested. This effect is separate from, and possibly synergistic with, the previously reported beneficial effects on intestinal flora reported for mannan oligosaccharides (Rozeboom et al., 2005) as the test was performed only with soluble metabolites extracted from fully fermented yeast culture without cell wall components of yeast.

\section{REFERENCES}

Barnes M.E., Durben D.J., Reeves S.G., Sanders R., 2006. Dietary yeast culture supplementation improves initial rearing of McConaughy strain rainbow trout. Aquacult. Nutr. 12, 388-394

Dann H.M., Drackley J.K., McCoy G.C., Hutjens M.F., Garrett J.E., 2000. Effects of yeast culture (Saccharomyces cerevisiae) on prepartum intake and postpartum intake and milk production of Jersey cows. J. Dairy Sci. 83, 123-127

Elad D., Brenner J., Markovitcs A., Yakobson B., Shlomovitz S., Basan J., 1998. Yeasts in the gastrointestinal tract of preweaned calves and possible involvement of Candida glabrata in neonatal calf diarrhea. Mycopathologia 141, 7-14

Kim S.W., Brandherm M., Freeland M., Newton B., Cook D., Yoon I., 2006. Yeast culture supplementation of gestation and lactation diets enhances litter weight gain of pigs. Proceedings of XIIth AAAP Animal Science Congress, Busan (Korea)

Kivaria F.M., Noordhuizen J.P.T.M., 2007. A retrospective study of the aetiology and temporal distribution of bovine clinical mastitis in smallholder dairy herds in the Dar es Salaam region of Tanzania. Vet. J. 173, 617-622

Liu Z.-B., Qi G.-H., Yoon I., 2002. Effect of yeast culture on production parameters and intestinal microflora in laying hens. Poultry Sci. 80, Suppl. 1, 89 (Abstr.)

Rozeboom D.W., Shaw D.T., Tempelman R.J., Miguel J.C., Pettigrew J.E., Connolly A., 2005. Effects of mannan oligosaccharide and an antimicrobial product in nursery diets on performance of pigs reared on three different farms. J. Anim. Sci. 83, 2637-2644

Schingoethe D.J., Linke K.N., Kalscheur K.F., Hippen A.R., Rennich D.R., Yoon I., 2004. Feed efficiency of mid-lactation dairy cows fed yeast culture during summer. J. Dairy Sci. 87, 41784181

Tangendjaja B., Yoon I., 2002. Effect of yeast culture on egg production and mortality in layer chickens. Poultry Sci. 80, Suppl. 1, 89 (Abstr.)

van den Bogaard A.E., Stobberingh E.E., 2000. Epidemiology of resistance to antibiotics. Links between animals and humans. Int. J. Antimicrob. Agents 14, 327-335

Witte W., 2000. Ecological impact of antibiotic use in animals on different complex microflora: environment. Int. J. Antimicrob. Agents 14, 321-325

Zlotowski P., Rozza D.B., Pescador C.A., Barcellos D.E., Ferreiro L., Sanches E.M., Driemeier D., 2006. Muco-cutaneous candidiasis in two pigs with postweaning multisystemic wasting syndrome. Vet. J. 171, 566-569 